\title{
Panorama del lenguaje y la literatura en las licenciaturas en Humanidades en Colombia
}

\author{
William Leonardo Perdomo Vanegas 1 \\ Leidy Yohanna López Pineda ${ }^{2}$ \\ María Fernanda Mahecha Oviedo ${ }^{3}$
}

Recibido: 10-08-2016

Aceptado: 18-10-2016

\section{RESUMEN}

El propósito de este artículo es presentar los resultados de la investigación "Marco referencial de la formación de docentes en el área de Humanidades y Lengua Castellana" en la que se llevó a cabo un estudio exploratorio sobre las tendencias y los referentes en torno a la formación de docentes en humanidades en las universidades colombianas. Para tal fin, la investigación se fundamentó en las prácticas pedagógicas, en los procesos de investigación, en las propuestas didácticas y en la fundamentación conceptual sobre el lenguaje y la literatura.

Palabras clave: semiótica, comunicación, educación, humanidades.

\section{Panorama of language and literature in the Degrees in Humanities in Colombia}

\begin{abstract}
The purpose of this article is to present the results of the investigation "Reference of teacher training in the area of Humanities and Spanish language" which was conducted an exploratory study on trends and

1. Doctor en literatura por la Universidad de Valladolid. Investigador titular de Ia Facultad de Educación la Corporación Universitaria Minuto de Dios.

Correo: wperdomo32@gmail.com

2. Licenciada en Humanidades. Líder del semillero de investigación en Literatura de la Facultad de Educación la Corporación Universitaria Minuto de Dios.

Correo: lilith131994@gmail.com

3. Licenciada en Humanidades. Líder del semillero de investigación en Creación Literaria de la Facultad de Educación la Corporación Universitaria Minuto de Dios.

Correo: fernandamahecha@gmail.com
\end{abstract}


referrers about teacher training in Humanities in Colombian universities. To this end, the research was based on pedagogical practices in research processes in the educational proposals and the conceptual foundation about language and literature.

Keywords: Semiotics; Comunication; Education; Humanities.

\section{Introducción}

La formación de docentes en Humanidades y Lengua Castellana en el contexto educativo colombiano se considera relevante y pertinente por las necesidades de formar en lectura y escritura $y$, de otro lado, por la pretensión de entender la comunicación como un mecanismo de consenso. En tal sentido, se hace pertinente realizar un estudio que contribuya a la revisión de los proyectos curriculares de las licenciaturas en esta área.

En esa medida, este documento tiene el propósito socializar los resultados de la investigación "Marco referencial de la formación de docentes en el área de Humanidades y Lengua Castellana" desarrollada por la Licenciatura en Humanidades y Facultad de Educación de la Corporación Universitaria Minuto de Dios. Uno de los propósitos de esta investigación es presentar las características y las tendencias en torno a la formación de docentes en el área de las Humanidades y lengua Castellana en Colombia. Para tal fin se analizaron los documentos de los proyectos curriculares y se prestó atención a las discusiones, las recomendaciones y los acuerdos de una serie de asesores y representantes de las diversas universidades con programas en Humanidades y Lengua Castellana o afines a nivel nacional.

La investigación se fundamentó en la revisión conceptual en torno a la lengua, el lenguaje y la literatura, puesto que estos son la base epistemológica para las Humanidades. En consecuencia, este artículo presenta el estado actual de los procesos académicos en las diferentes universidades a nivel nacional, así como un análisis descriptivo sobre los proyectos curriculares de laslicenciaturas en el área mencionada. Para ello, se hizo una revisión documental en torno a las propuestas de formación 
de docentes en varias instituciones educativas, específicamente se analizaron los planes de estudios, los proyectos curriculares y educativo de 41 programas académicos del país.

Por tal motivo, este estudio permite, en primera instancia, reflexionar sobre la pertinencia del currículo de Humanidades y Lengua Castellana en relación con los nuevos Lineamientos de calidad para las licenciaturas en educación (2015) y, en segundo lugar, reflexionar sobre la fundamentación epistemológica, las propuestas didácticas y las prácticas pedagógicas en coherencia con los retos que surgen en la actualidad.

\section{Estudio descriptivo sobre la formación en Humanidades y Lengua Castellana}

En el desarrollo del presente estudio se revisaron las universidades colombianas que ofrecen licenciaturas relacionadas con las Humanidades, la Lengua Castellana o española, la Literatura y las Lenguas Modernas. En ese sentido, se descartaron los programas académicos que se centran directamente en los estudios literarios y las ciencias del lenguaje, puesto que su misión no se enfatiza en el ámbito educativo. Así, se seleccionaron cuarenta y un (42) licenciaturas activas en el 2015 en el país.

De acuerdo con el Sistema Nacional de Información de la Educación Superior - SNIES -, que es un sistema creado para responder a las necesidades de información de la educación superior en Colombia, en el año 2015, a nivel nacional se podía encontrar dos grupos de programas que, acorde con su denominación, se relacionaban con las Humanidades, la Lengua Castellana y el Lenguaje. En el primer grupo se encuentran las licenciaturas en educación básica con énfasis (Ver tabla \# 1). 


\begin{tabular}{|c|c|c|}
\hline Universidad & Denominación del programa & Créditos \\
\hline $\begin{array}{l}\text { Corporación Universitaria del } \\
\text { Caribe (Distancia) }\end{array}$ & $\begin{array}{l}\text { Licenciatura en Educación Básica con Énfasis } \\
\text { en Humanidades }\end{array}$ & 153 \\
\hline Universidad de Córdoba & $\begin{array}{l}\text { Licenciatura en Educación Básica con Énfasis } \\
\text { en Humanidades - Lengua Castellana }\end{array}$ & 154 \\
\hline Universidad de Nariño & $\begin{array}{l}\text { Licenciatura en Educación Básica con Énfasis } \\
\text { en Humanidades Lengua Castellana e Inglés }\end{array}$ & 149 \\
\hline $\begin{array}{l}\text { Universidad Distrital - Francisco } \\
\text { José de Caldas }\end{array}$ & $\begin{array}{l}\text { Licenciatura en educación básica con énfasis } \\
\text { en Humanidades y Lengua Castellana }\end{array}$ & 173 \\
\hline $\begin{array}{l}\text { Universidad del Magdalena } \\
\text { (Distancia) }\end{array}$ & $\begin{array}{l}\text { Licenciatura en Educación Básica con Énfasis } \\
\text { en Humanidades y Lengua Castellana }\end{array}$ & 145 \\
\hline $\begin{array}{l}\text { Universidad Industrial de } \\
\text { Santander - UIS }\end{array}$ & $\begin{array}{l}\text { Licenciatura en Educación Básica con Énfasis } \\
\text { en Lengua Castellana }\end{array}$ & 170 \\
\hline $\begin{array}{l}\text { Universidad Pedagógica y } \\
\text { Tecnológica de Colombia }\end{array}$ & $\begin{array}{l}\text { Licenciatura en Educación Básica con Énfasis } \\
\text { en Matemáticas y Español }\end{array}$ & 167 \\
\hline Universidad de Antioquia & $\begin{array}{l}\text { Licenciatura en Educación Básica con Énfasis } \\
\text { en Humanidades y Lengua Castellana }\end{array}$ & 177 \\
\hline $\begin{array}{l}\text { Corporación Universitaria } \\
\text { Adventista }\end{array}$ & $\begin{array}{l}\text { Licenciatura en Educación Básica con Énfasis } \\
\text { en Humanidades: Español e Inglés }\end{array}$ & 144 \\
\hline $\begin{array}{l}\text { Fundación Universitaria } \\
\text { Monserrate }\end{array}$ & $\begin{array}{l}\text { Licenciatura en Educación Básica con Énfasis } \\
\text { en Lengua Castellana }\end{array}$ & 144 \\
\hline $\begin{array}{l}\text { Universidad Tecnológica de } \\
\text { Antioquia }\end{array}$ & $\begin{array}{l}\text { Licenciatura en Educación Básica con Énfasis } \\
\text { en Humanidades y Lengua Castellana }\end{array}$ & 168 \\
\hline Universidad de Cundinamarca & $\begin{array}{l}\text { Licenciatura en Educación Básica con Énfasis } \\
\text { en Humanidades y Lengua Castellana e Inglés }\end{array}$ & 158 \\
\hline $\begin{array}{l}\text { Corporación Universitaria } \\
\text { Minuto de Dios }\end{array}$ & $\begin{array}{l}\text { Licenciatura en Educación Básica con Énfasis } \\
\text { en Humanidades y Lengua Castellana }\end{array}$ & 144 \\
\hline Universidad INCCA & $\begin{array}{l}\text { Licenciatura en Educación Básica con Énfasis } \\
\text { en Humanidades y Lengua Castellana e Inglés }\end{array}$ & 165 \\
\hline $\begin{array}{l}\text { Universidad Pedagógica } \\
\text { Nacional }\end{array}$ & $\begin{array}{l}\text { Licenciatura en Educación Básica con Énfasis } \\
\text { en Humanidades y Lengua Castellana e Inglés }\end{array}$ & 160 \\
\hline $\begin{array}{l}\text { Universidad Pedagógica } \\
\text { Nacional }\end{array}$ & $\begin{array}{l}\text { Licenciatura en Educación Básica con Énfasis } \\
\text { en Humanidades, Lengua Castellana y } \\
\text { Lenguas }\end{array}$ & 160 \\
\hline Universidad Libre (Bogotá) & $\begin{array}{l}\text { Licenciatura en Educación Básica con Énfasis } \\
\text { en Humanidades e Idiomas }\end{array}$ & 160 \\
\hline Universidad Libre (Socorro) & $\begin{array}{l}\text { Licenciatura en Educación Básica con Énfasis } \\
\text { en Humanidades y Lenguas }\end{array}$ & 160 \\
\hline
\end{tabular}

Tabla No. 1: Programas de Licenciatura en educación básica con énfasis

El segundo grupo es el de las licenciaturas que no hacen alusión a la educación básica, aunque sí se relacionan con las Humanidades, la Lengua Castellana, el Lenguaje o la Literatura (Ver tabla \# 2. Programas que descartan la educación básica en su denominación). 


\begin{tabular}{|c|c|c|}
\hline Universidad & Denominación del programa & Créditos \\
\hline Universidad de la Amazonía & Licenciatura en Lengua Castellana y Literatura & 131 \\
\hline $\begin{array}{l}\text { Corporación Universitaria del } \\
\text { Caribe (Presencial) }\end{array}$ & Licenciatura en Literatura y lengua Castellana & 155 \\
\hline Universidad de Nariño & Licenciatura en Lengua Castellana y Literatura & 10 sem. \\
\hline Universidad de Pamplona & $\begin{array}{l}\text { Licenciatura en Lengua Castellana y } \\
\text { Comunicación }\end{array}$ & 162 \\
\hline $\begin{array}{l}\text { Universidad de San } \\
\text { Buenaventura (Cali) }\end{array}$ & Licenciatura en Lengua Castellana & 153 \\
\hline $\begin{array}{l}\text { Universidad Industrial de } \\
\text { Santander - UIS }\end{array}$ & Licenciatura en Español y Literatura & 170 \\
\hline Universidad Popular del Cesar & Licenciatura en Lengua Castellana e Inglés & 170 \\
\hline Universidad del Valle & Licenciatura en Literatura & 171 \\
\hline Universidad del Quindío & Licenciatura en Español y Literatura & 159 \\
\hline $\begin{array}{l}\text { Universidad Tecnológica de } \\
\text { Pereira }\end{array}$ & Licenciatura en Español y Literatura & 152 \\
\hline Universidad del Atlántico & Licenciatura en Español y Literatura & 140 \\
\hline Universidad Santo Tomas & Licenciatura en Filosofía y Lengua Castellana & 158 \\
\hline Universidad del Cauca & Licenciatura en Español e Inglés & 173 \\
\hline Universidad del Tolima & Licenciatura en Lengua Castellana & 170 \\
\hline Universidad Católica del Norte & Licenciatura en Lengua Castellana & 136 \\
\hline $\begin{array}{l}\text { Fundación Universitaria Luis } \\
\text { Amigó }\end{array}$ & Licenciatura en Lengua Castellana (Distancia) & 144 \\
\hline Universidad La Gran Colombia & Licenciatura en Lingüística y Literatura & 155 \\
\hline Universidad Sur Colombiana & Licenciatura en Lengua Castellana & 160 \\
\hline Universidad Antonio Nariño & Licenciatura en Lengua Castellana e Inglés & 144 \\
\hline $\begin{array}{l}\text { Universidad Cooperativa de } \\
\text { Colombia (Bucaramanga) }\end{array}$ & Licenciatura en Lengua Castellana e Inglés & 160 \\
\hline Universidad de la Salle & $\begin{array}{l}\text { Licenciatura en Lengua Castellana, Inglés y } \\
\text { Francés }\end{array}$ & 180 \\
\hline Universidad Javeriana & Licenciatura en Lenguas Modernas & 180 \\
\hline Universidad Sergio Arboleda & Licenciatura en Filosofía y Humanidades & 160 \\
\hline $\begin{array}{l}\text { Universidad Tecnológica del } \\
\text { Chocó }\end{array}$ & Licenciatura en Lingüística y literatura & 147 \\
\hline
\end{tabular}

Tabla No. 2: Programas que descartan la educación básica en su denominación

Al realizar una revisión sobre las universidades que ofrecen programas en educación en Lengua Castellana en la actualidad, se evidencia que un gran número de programas ha optado por obviar educación básica para denominar el programa en relación directa con el área disciplinar. Además, se puede observar la diversidad en relación con el número de 
créditos ofertados, la Universidad de la Amazonía oferta un programa de 131 créditos en contraste, universidades como la Javeriana y la Salle ofrecen programas de 180 créditos.

De acuerdo con el SNIES (2015), en Colombia hay registradas dieciocho (18) programas que ofrecen la licenciatura en Educación básica con algún tipo de énfasis relacionado con las Humanidades o la Lengua Castellana, entre estas, siete (7) vinculan las Humanidades o la Lengua castellana con inglés u otro idioma extranjero. Además se encuentra una (1) licenciatura que menciona en su denominación las Matemáticas. También existen veintitrés (23) que no consideran en su titulación el campo de desempeño de la educación básica, entre estas una (1) relaciona la Filosofía con las Humanidades y otra (1) con la Comunicación.

\section{Tendencias de la formación en Humanidades}

En el marco de este proceso investigativo, que abordó como objeto de estudio las propuestas curriculares a nivel nacional en torno a la formación en el área de Humanidades, es indispensable definir la fundamentación conceptual y la metodología empleadas para la interpretación y la comprensión de estos procesos. En ese orden de ideas, el estudio definió las siguientes categorías para llevar a cabo el análisis de los proyectos curriculares y educativos de los programas académicos en Humanidades: fundamentación conceptual respecto al lenguaje y la lengua, la concepción de literatura, el acercamiento a otros sistemas simbólicos abordados por la semiótica, la incursión de los medios de comunicación, la proposición de didácticas en torno a la lengua y la coherencia de las prácticas pedagógicas con la implementación de procesos investigativos. En coherencia con esta fundamentación, los estudios hermenéuticos también contribuyeron a la revisión de los documentos y archivos obtenidos en los sitios web de cada universidad y algunos otros facilitados por colaboradores de las mismas instituciones.

Así, se delimitó la investigación en un enfoque y diseño cualitativos, que privilegian el análisis del objeto de estudio y sus particularidades. En palabras de Alejandra Reguera, la investigación cualitativa describe fenómenos que ocurren en un contexto social y cultural, pero no tiene la necesidad de llevarlos al plano de la experimentación (2008, p. 78). En ese mismo sentido, Bonilla y Rodríguez afirman que la investigación 
cualitativa es "aquella que pretende cualificar y describir el fenómeno social a partir de los rasgos determinantes, percibidos desde elementos que están dentro de la situación estudiada" (2000, p. 68).

Por otra parte, esta investigación se fundamentó en los estudios hermenéuticos debido a la naturaleza social del objeto de estudio y a los procedimientos aplicados en el análisis del mismo. Estos estudios, relacionados con las ciencias humanas y sociales, proveen los medios pertinentes para alcanzar la interpretación del fenómeno estudiado, afrontando las dificultades que surgen de la complejidad del lenguaje.

En relación con lo anterior, este estudio analítico buscó "familiarizarse con un fenómeno social y adquirir nuevos conocimientos sobre el mismo con el fin de formular un problema más preciso" (Reguera, 2008, p. 45), también se centró en realizar un análisis hermenéutico desde la praxis investigativa. Para tal efecto se consideraron los elementos fundamentales expuestos por Manuel Baeza, quien afirma que la lectura hermenéutica contempla los siguientes aspectos: el conocimiento del contexto, elección del corpus, creación de las categorías de interpretación, análisis del corpus y la presentación de las conclusiones (Baeza, 2002, p. 163-164). Por lo tanto, centrarse en un objeto de estudio con tales características requirió de seis etapas básicas para lograr su objetivo, estas fases parten del diseño de la investigación cualitativa y son coherentes con los estudios hermenéuticos propuesto por Baeza (2002).

La primera etapa fue la identificación del problema de investigación en la que se propuso, explícita y sistemáticamente, construir conocimiento o crear un espacio de reflexión sobre la comprensión de los documentos que conforman la propuesta curricular de cada programa académico. La siguiente etapa se centró en la presentación de una propuesta metodológica que parte, en primer lugar, del problema construido, es decir, dependía de los objetivos específicos de la investigación. En la etapa a seguir se llevó a cabo el proceso de documentación en el que se recogió la información pertinente en torno a los aspectos teóricoconceptuales. En cuarto lugar, se propuso un modelo estructurado con base en una serie de categorías que fundamentaron la interpretación de los proyectos curriculares. Posteriormente, se realizó el análisis, a partir de una lectura hermenéutica, de las propuestas académicas para llegar a una comprensión y, finalmente, se socializaron los hallazgos obtenidos a partir de los objetivos planteados (Bonilla y Rodríguez, 2000, p. 72). 
Por consiguiente, el análisis de los documentos curriculares se consolidó como un proceso investigativo enmarcado en el enfoque y el diseño cualitativo, que abordó un fenómeno social a partir de un estudio hermenéutico para el análisis. De acuerdo con la perspectiva metodológica de esta investigación las técnicas de recolección de información propuestas fueron las siguientes: revisión de las Políticas Educativas Nacionales sobre la formación de docentes en el área de humanidades, análisis de los currículos en el área de Humanidades y Lengua Castellana (Proyectos curriculares de las universidades nacionales), discusión e interpretación de resultados con pares colaborativos de las universidades nacionales y socialización de los resultados a la comunidad académica.

En esa medida, entre las primeras tendencias se evidencia que la mayoría de los programas justifican su denominación con base en las Políticas Nacionales de Educación, específicamente en la Ley 30 de 1992, la Ley 115 de 1994 y en el Decreto 272 de 1998. Se puede afirmar que estas licenciaturas tienen en común acuerdo formar profesionales de la educación en el campo de las Humanidades y la Lengua Castellana, relacionadas, a su vez, con las Lenguas Modernas, la Literatura, la Comunicación y la Filosofía.

Además de ello, estos programas académicos apuestan poruna formación en competencias en torno al quehacer pedagógico, investigativo y comunicativo, que contribuya al desarrollo social y cultural de la comunidad, así como a la reflexión en torno a los procesos didácticos en el contexto educativo colombiano. Por lo tanto, desde los objetivos de los proyectos curriculares se evidencia una tendencia por un desarrollo académico que integre la reflexión, la investigación y la innovación sobre los procesos de enseñanza y aprendizaje de la lengua castellana. Adicionalmente, las universidades de esencia religiosa hacen énfasis en formar un profesional de la educación de acuerdo con los principios católicos, así como todas las universidades cuentan con un componente que promueva la filosofía o principios humanistas de la institución.

De otro lado, las propuestas curriculares de las Licenciaturas en Humanidades estructuran sus planes de estudio por componentes, entre ellos se encuentra uno relacionado con pedagógico general o básico, otro con la práctica pedagógica, uno más referido a lo disciplinar y uno último que enmarca el carácter ético del currículo. Estos componentes sustentan la flexibilidad, la interdisciplinariedad, la integralidad y la transdisciplinariedad del programa, así como contribuyen a proponer 
los núcleos del saber pedagógico determinados por la Resolución 272 y; además, respaldan la fundamentación epistemológica y conceptual de cada licenciatura.

\section{La lengua y el lenguaje en los programas de licenciatura en Humanidades}

El lenguaje es un fenómeno complejo y, por ello, cualquier definición que lo presente de un modo simplista no conseguirá más que viciarlo y corromperlo. En palabras de Edgar Morin, el lenguaje es un fenómeno complejo no simplificable que forma parte tanto de la realidad inmediata como de la más abstracta (2005, p. 22).

Al ser un fenómeno complejo, no hay una definición unívoca del lenguaje. Por su carácter multidimensional, los intentos de acercamiento a esta noción son parciales y no totales. Como objeto propio de las ciencias humanas, "el lenguaje es entonces, una palabra problema y no una palabra solución" (Morin, 2005, p. 22). Por si eso fuera poco, el lenguaje es un fenómeno mutante, que se reproduce y se despliega en multitud de posibilidades y versiones, con lo que la definición que se le conceda hoy, no es definitiva.

La preocupación por definir el concepto de lenguaje es histórica. Preocupación que alcanzó la cumbre en el siglo XX, por el interés que desde diferentes áreas de conocimiento, como la Filosofía o las Ciencias Sociales suscitó el estudio del lenguaje. Una revisión histórica de este interés evidencia que no hay un solo filósofo que no haya dedicado atención en su obra al problema del lenguaje: en la Época Clásica, Platón y Aristóteles, pasando, por supuesto, por los presocráticos. En la Edad Media, gran parte de los estudiosos escribieron acerca del lenguaje, destacándose Santo Tomás y San Agustín. En la Época Moderna, con el surgimiento de la ciencia del empirismo y la razón, también existe una filosofía del lenguaje. Pero es en la Época Contemporánea cuando se fortalecen todas las disciplinas que hoy, desde uno $u$ otro interés, tienen al lenguaje como objeto de estudio: la semántica, la sintaxis, la pragmática, la hermenéutica, el análisis lingüístico, el estructuralismo, la filología, la semiótica, la lingüística del texto y los estudios del discurso, entre otras (Soriano y otros, 2014, p. 91). 
El lenguaje hoy se manifiesta en todas sus opciones, por lo que se habla de lenguaje virtual, audiovisual, gestual, escénico, matemático, entre otros. La realidad que se presenta en la actualidad es la del lenguaje que desborda la red del logos en que se hallaba retenido, para manifestarse en toda su variabilidad. El logocentrismo le da paso al iconocentrismo para abordar el lenguaje en cualquiera de sus manifestaciones sin reducciones. En esa medida, una profundización en el fenómeno del lenguaje, como fenómeno complejo, más allá de su versión puramente verbal permitirá comprender mejor el contexto social. Por consiguiente, el lenguaje es uno de los aspectos más relevantes de la sociedad, la cultura y el individuo.

Al respecto, José Antonio Marina plantea que el lenguaje nace en el mundo de la vida y tiene una función práctica: Comunicar, organizar la colaboración, pedir, transferir conocimientos, planificar y dirigir la conducta. Sirve para la comunicación exterior y para la construcción del propio sujeto (1999, p. 43).

En relación con el lenguaje y la lengua, gran parte de los currículos optan por una formación teórica de la lingüística y la literatura, con su respectivo sustento filosófico, psicológico y biológico. En este sentido, los proyectos curriculares proponen relaciones entre la lengua y su uso en el contexto social desde el ámbito verbal y no verbal; igualmente, se hace énfasis en las ciencias del lenguaje y sus vertientes como la psicolingüística, la sociolingüística y el análisis del discurso.

De otra parte, se observa una aproximación hacia los aspectos formales de la lengua, como la gramática, la sintaxis, la fonología y la ortografía; sin embargo, se privilegia una perspectiva discursiva. En esa medida, se evidencia una enseñanza de la lengua que pretende trascender del desarrollo de las habilidades lingüísticas para acercarse a las funciones de la lengua en un contexto social. Se propone abordar el lenguaje desde el proceso de significación, entendido como el nivel que permite otorgar cierta categoría de signo a los fenómenos sociales y a los discursos presentes en la interacción cultural.

\section{Literatura en la formación de docentes}

Actualmente la definición de literatura se relaciona con la cultura y la sociedad y, al mismo tiempo, designa tres acciones específicas. En 
primer lugar, la literatura se define como la práctica de creación verbal, que involucra la producción de obras escritas por ciertos individuos que buscan valorar la sociedad en la que están inmersos; en segundo lugar, se comprende como la actividad de investigación y conocimiento de dicha práctica verbal, que se define como la labor del crítico o estudioso del proceso de producción de las distintas manifestaciones literarias; y por último, la literatura se entiende como la práctica institucional educativa que socialmente permite producir y conservar los productos de la creación verbal considerados como componentes esenciales del patrimonio cultural. Aquí también se vincula lo que se denomina didáctica de la literatura (Huaman, 2007).

En este sentido, esta área está conformada por tres elementos: la historia de la literatura, que estudia el origen y la evolución de la literatura; la crítica literaria, que estudia la valoración de la historia literaria; y la teoría literaria, que abarca el estudio de los principios conceptuales de las manifestaciones literarias (Gil-Albarellos, 2006, p. 24).

En el análisis realizado en los programas de Humanidades se puede observar que no todos desarrollan un componente sobre la literatura. Se presentan espacios relacionados con las literaturas universales, nacionales y regionales, sin certificar una reflexión en torno a la práctica pedagógica desde el lenguaje poético. En esa misma medida, se evidencia que las tendencias en la formación de profesores en el campo de la literatura privilegian la lectura y la comprensión de los textos literarios, así como las teorías y metodologías de los enfoques semiótico y hermenéutico. Se incluyen también los aportes desde la recepción literaria, al igual que estrategias para la animación en lectura y literatura.

Por otra parte, un aspecto para resaltar en algunos planes de estudio es ofrecer cursos que se relacionen con la literatura de la región. Tal es el caso de la Universidad Tecnológica del Chocó con Literatura Afrocolombiana, la Universidad del Valle ofrece electivas como Literatura Regional Vallecaucana, Literatura Indígena y Literatura Indígena Contemporánea, por su parte, la Universidad de Córdoba ofrece Literatura de Córdoba y Literatura del Caribe, este espacio académico también lo ofrece la Universidad del Atlántico; por último, la Corporación Universitaria del Caribe -CECAR- ofrece Literatura Regional Costeña.

Así mismo, todos los programas sustentan sus propuestas curriculares con base en una formación integral retomando la literatura, puesto que 
esta se puede relacionar con un sin número de aspectos académicos en la escuela. Por otra parte, algunas universidades resaltan la importancia de un espacio académico donde se trabaje la didáctica de la literatura y la teoría literaria.

De otro lado, no son muchos los programas que se refieren abiertamente a un componente comunicativo, no obstante, gran parte de las propuestas optan por una formación de docentes que comprendan los fenómenos comunicativos y su impacto en el contexto social; de igual manera, se hace alusión a la importancia de estudiar las ideas y las valoraciones que se divulgan a través de los medios y las relaciones de poder que se vehiculan a través de ellos.

En ese sentido, todas las universidades se comprometen a generar en los futuros docentes la habilidad comunicativa que le permita al mismo construir canales asequibles entre el conocimiento y la práctica, entre el saber y las necesidades sociales, entre las características de los sujetos y el saber a construir. En otras palabras, se defiende una formación que fomente la construcción de habilidades comunicativas que no se restrinjan al dominio de las didácticas, sino a la comprensión del sujeto, del otro y de lo otro. Siendo así, los espacios académicos que se relacionan con este componente son aquellos donde convergen los saberes dedicados a la reflexión de los fenómenos comunicativos, sus transformaciones e implicaciones en el ámbito cultural y subjetivo del hombre.

Estos espacios se presentan como estrategias que acercan a los estudiantes a los nuevos lenguajes y las formas de comunicación emergentes que implican nuevos retos para la educación básica y media; también se menciona la importancia de la reflexión, por parte de los educadores, sobre la función de la imagen y los discursos multimodales en los actuales contextos socio-culturales. De igual manera, se alude al uso reflexivo y creativo de las tecnologías de la información y la comunicación, las cuales generan nuevos horizontes y modos de interacción entre los sujetos y la sociedad.

Desde esta perspectiva, los espacios académicos que evidencian alguna relación con la comunicación se justifican a partir de la interdisciplinariedad, en la medida que estimulan la investigación y la indagación a la luz de cuestionamientos recientes suscitados por el influjo de los signos y las tecnologías en la cotidianidad. Por consiguiente, los programas conciben la comunicación como una compleja red de 
interacciones sociales que estimula el intercambio de saberes y visiones que se enfrentan constantemente para contribuir a la crítica, la autocrítica y la creatividad.

Dado su carácter social y sígnico, en algunos programas el ámbito de la comunicación se presenta al interior del componente semiótico $y_{\text {, }}$ también, se sustenta en aras de lograr una mejor interpretación de todos los sistemas de comunicación dispuestos para la representación, la comunicación y la construcción de la realidad en el contexto actual.

En definitiva, los proyectos curriculares se definen como estrategias de desarrollo integral al relacionar en sus planes de estudio los espacios de lenguaje, literatura y comunicación; sin embargo, en el análisis se observa que la mayoría de los programas dan prioridad a los cursos relacionados con la lengua, el lenguaje y la literatura, dejando en tercer lugar las asignaturas de comunicación y semiótica.

\begin{tabular}{|c|c|c|c|}
\hline Universidad & Lingüística & Literatura & Comunicación \\
\hline Universidad Tecnológica de Chocó & 16 & 11 & 4 \\
\hline Universidad de Córdoba & 8 & 9 & 1 \\
\hline Universidad de Nariño & 9 & 4 & 0 \\
\hline $\begin{array}{l}\text { Universidad de Nariño - Lic. Lengua } \\
\text { Castellana y Literatura }\end{array}$ & 6 & 6 & 1 \\
\hline $\begin{array}{l}\text { Universidad Distrital - Francisco José de } \\
\text { Caldas }\end{array}$ & 10 & 7 & 1 \\
\hline Universidad del Magdalena (Distancia) & 7 & 7 & 1 \\
\hline Universidad Industrial de Santander - UIS & 8 & 5 & 1 \\
\hline $\begin{array}{l}\text { Universidad Pedagógica y Tecnológica de } \\
\text { Colombia }\end{array}$ & 3 & 0 & 0 \\
\hline Universidad de Antioquia & 9 & 12 & 1 \\
\hline Corporación Universitaria Adventista & 4 & 3 & 1 \\
\hline Fundación Universitaria Monserrate & 6 & 7 & 1 \\
\hline Universidad Tecnológica de Antioquia & 6 & 7 & 1 \\
\hline Universidad de Cundinamarca & - & - & - \\
\hline Corporación Universitaria Minuto de Dios & 5 & 10 & 1 \\
\hline Universidad INCCA & 6 & 8 & 0 \\
\hline Universidad Pedagógica Nacional & 8 & 7 & 5 \\
\hline Universidad Pedagógica Nacional & 7 & 7 & 5 \\
\hline Universidad Libre (Bogotá) & 1 & 7 & 1 \\
\hline Universidad Libre (Socorro) & 7 & 8 & 0 \\
\hline Universidad de Amazonía & 7 & 12 & 4 \\
\hline
\end{tabular}




\begin{tabular}{|c|c|c|c|}
\hline $\begin{array}{l}\text { Corporación Universitaria del Caribe } \\
\text { (Presencial) }\end{array}$ & 10 & 6 & 1 \\
\hline Universidad de Pamplona & 12 & 8 & 1 \\
\hline Universidad de San Buenaventura (Cali) & 6 & 9 & 1 \\
\hline Universidad Industrial de Santander - UIS & 7 & 9 & 1 \\
\hline Universidad Popular del Cesar & - & - & - \\
\hline Universidad del Valle & 0 & 26 & 0 \\
\hline Universidad del Quindío & - & - & - \\
\hline Universidad Tecnológica de Pereira & 10 & 12 & 1 \\
\hline Universidad del Atlántico & 11 & 13 & 1 \\
\hline Universidad Santo Tomas & 5 & 5 & 2 \\
\hline Universidad del Cauca & & 1 & \\
\hline Universidad del Tolima & 6 & 12 & 0 \\
\hline Universidad Católica del Norte & 3 & 1 & 1 \\
\hline Fundación Universitaria Luis Amigó & 10 & 7 & 2 \\
\hline Universidad La Gran Colombia & 9 & 10 & 0 \\
\hline Universidad Sur Colombiana & - & - & - \\
\hline Universidad Antonio Nariño & - & - & - \\
\hline $\begin{array}{l}\text { Universidad Cooperativa de Colombia } \\
\text { (Bucaramanga) }\end{array}$ & - & - & - \\
\hline Universidad de la Salle & 5 & 0 & 1 \\
\hline Universidad Javeriana & 8 & 0 & 0 \\
\hline Universidad Sergio Arboleda & 3 & 4 & 0 \\
\hline $\begin{array}{l}\text { Corporación Universitaria del Caribe } \\
\text { (Distancia) }\end{array}$ & 8 & 5 & 1 \\
\hline
\end{tabular}

Tabla No. 3: Relación de los espacios académicos referidos al lenguaje, la literatura y la comunicación.

En el cuadro se observa la distribución de las instituciones respecto a la formación en lingüística, literatura y comunicación. Así, se puede afirmar que La UniversidadTecnológica del Chocó cuenta con más espacios para la formación en lingüística, dieciséis (16); sin embargo, en literatura cuenta con once (11) espacios. Por otra parte, la Universidad de Pamplona da prioridad a la formación en lingüística con doce espacios (12), a la parte de literatura le dedica ocho (8) espacios y a la parte de comunicación un (1) espacio. De otro lado, la Universidad de la Amazonía dedica doce (12) espacios a la literatura, siete (7) a la parte de lingüística y cuatro (4) a la parte de comunicación. Para finalizar, se puede concluir que los aspectos de formación se encuentran divididos entre la formación en lingüística y literatura, sin embargo, es notable el poco espacio otorgado a los cursos de comunicación, ya que los tres ámbitos son relevantes al hablar de formación integra 


\section{Procesos de investigación en las licenciaturas de Humanidades}

En el caso concreto de la investigación en el marco de las licenciaturas en Humanidades y Lengua castellana, los procesos investigativos se centran en la revisión de los currículos desde los avances logrados en los procesos investigativos y de proyección social; esto es más evidente cuando se entiende que los profesionales docentes y los estudiantes que hacen parte de los semilleros y procesos de prácticas en responsabilidad social y profesionales retoman sus experiencias y las llevan al aula de clase y a los comités curriculares.

Desde este mismo marco, se evidencian acciones que buscan la aplicación y socialización de algún modelo pedagógico, lo que lleva a impregnar todas las acciones. Muchos de estos procesos de investigación y muchas prácticas profesionales se realizan desde el enfoque pedagógico y así se contribuyen a expandirlo a todos los procesos de la institución.

De otro lado, estos procesos conllevan a realizar esfuerzos por publicar el fruto de sus trabajos investigativos y de proyección social, a través de sistematización de experiencias e investigación básica, situada y aplicada. Estas publicaciones, sean impresas o virtuales, terminan enriqueciendo la formación integral de los estudiantes y el quehacer de los docentes.

De igual manera, es común ver que las instituciones educativas centran gran parte de la labor de indagación en la investigación formativa, la cual se entiende como los diferentes ambientes de aprendizaje adecuados para la generación de una cultura de la investigación en la comunidad académica, reconociendo que el aprendizaje es un proceso de construcción del conocimiento y la enseñanza es el objeto de la reflexión desde la práctica.

En relación con la investigación y las prácticas, se podrían describir tres características de las propuestas curriculares. La primera resalta las universidades que ofrecen los espacios académicos de investigación y prácticas pedagógicas antes de la mitad del plan de estudios, en segundo lugar se evidencian los programas que proponen estos espacios después de cursar el $50 \%$ del mismo. En tercer lugar se puede hablar de los programas que cuentan con una línea o grupo de investigación centrado en las didácticas. 
En términos generales, las licenciaturas proponen desarrollar competencias investigativas desde el currículo y en relación con las prácticas, a través de los espacios académicos con énfasis explícitamente formativo en investigación, formación artística y cultural; además, los diferentes programas fomentan estrategias, metodologías, principios y conceptos fundamentales. Así mismo, la investigación se consolida al interior de las licenciaturas por medio de las líneas de investigación, las cuales están soportadas en los proyectos investigativos que surgen en los programas académicos de las facultades y que señalan la ruta hacia donde se debe enfocar el proceso de investigación formativa.

En definitiva, los procesos investigativos de las universidades tienden a responder a las necesidades del desarrollo humano y social, a las experiencias propias de los estudiantes en los ámbitos educativos y a las tendencias nacionales e internacionales sobre generación de nuevo conocimiento; por ello gran parte de los trabajos de investigación se inscriben en las diversas regiones donde existen necesidades sociales y se pueda entrar a interactuar, dinamizar y contribuir a la construcción de una nueva sociedad.

\section{Conclusiones del estudio}

Las propuestas curriculares de formación de profesores en Humanidades y Lengua Castellana hacen un especial énfasis en el desarrollo humano y social de la persona basándose en criterios como la flexibilidad, la interdisciplinariedad, la integralidad y la transdisciplinariedad. Ello supone un desarrollo humanista que gira en torno a la construcción permanente, a partir de las relaciones que establece con la cultura y, por tanto, con el entorno social, lo que implica que el proceso de aprendizaje de los futuros educadores sea constante y se centre en las relaciones con los otros.

Por consiguiente, se observa que todas las licenciaturas responden a los criterios curriculares propuestos por el Consejo Nacional de Acreditación - CNA - en cuanto a que los proyectos curriculares proponen una formación integral basada en el compromiso social y pedagógico. Estos se fundamentan en el principio de desarrollar un proyecto educativo que posibilite la convivencia y facilite el aprendizaje por medio de la significación. En esa medida, se evidencia que en la mayoría de los 
programas responden a las directrices de las políticas nacionales, en las que se pretende aunar esfuerzos para ofrecer una formación que integre la reflexión, la investigación y la innovación sobre los procesos de enseñanza y aprendizaje de la Lengua Castellana y los diversos lenguajes.

En consecuencia, los programas se justifican en una demanda social de profesionales en el área de las Humanidades que orienten y mejoren los procesos de socialización y aprendizaje, pues son estos la base de la interacción del hombre como ser social y de su educación. Así, en espacios de interacción con comunidades, como el propiciado por las prácticas sociales y las profesionales, los futuros licenciados desarrollan procesos comunicativos, lingüísticos, literarios y semióticos que llevan a los grupos a valorar la lengua y la diversidad lingüística, a reflexionar y criticar los discursos mediáticos, a interpretar las relaciones entre la creación literaria y poética y las sociedades y a comprender mejor un sinnúmero de códigos que, aunque no se basen en palabras, desbordan el sentido social.

Por ende, desde las propuestas curriculares, dicha función social de la literatura y el lenguaje resuelve una necesidad social de la comunidad, la de leer, escribir, hablar y escuchar desde los códigos precisos de la lengua, desde lo impreciso de los lenguajes no verbales, y en función de los criterios de adecuación y usos del lenguaje que llevan a los sujetos sociales a comunicarse, representar, construir y transformar la realidad social.

Respecto a la literatura, las licenciaturas en general ofrecen espacios académicos dedicados a la formación de la misma. La intensidad horaria de estos espacios varía de acuerdo a la denominación y objeto de estudio de la licenciatura, cuando es solo literatura su plan de estudios se enfoca al estudio de la misma desde diversos componentes y asignaturas que la abarcan desde diferentes aspectos. No obstante, cuando las licenciaturas tienen más de un objeto de estudio como es el caso de las licenciaturas con énfasis en Humanidades y Lengua Castellana, Comunicación, Idioma Extranjero o Filosofía, la parte de la literatura tiene un espacio más reducido para su estudio. Sin embargo, se expresa la necesidad de que un futuro licenciado conozca las perspectivas generales que ofrecen los estudios de la literatura.

De otro lado, no en todos los proyectos curriculares se estructuran con un componente semiótico - comunicativo, sin embargo sí es evidente 
la intensión de formar docentes desde este enfoque que contribuya a la reflexión sobre los fenómenos sociales, tal como lo expresan las Políticas Nacionales de Educación. En esa misma medida, se evidencia una tendencia a crear procesos académicos relacionados con las habilidades comunicativas que contribuyan a la formación integral.

Por otra parte, en los proyectos curriculares se reconoce la importancia de la didáctica en cada una de las licenciaturas, puesto que se enfatiza en la necesidad de crear metodologías y materiales que faciliten el desarrollo de las competencias en los educandos. Teniendo en cuenta el balance de la didáctica en las universidades revisadas, es evidente la relevancia que se le da a espacios en los que se abordan mecanismos y herramientas para enseñar la lengua y la literatura; sin embargo, en el estudio también se evidencia que los espacios destinados a las didácticas son pocos en relación con los cursos disciplinares, más aún cuando los currículos se definen como procesos de formación integral. De igual forma, la investigación en el campo de la didáctica aporta al desarrollo de procesos educativos que fortalecen la enseñanza de la lengua.

Las licenciaturas optan por desarrollar procesos de investigación relacionados con la práctica educativa por medio de espacios específicos en el área; no obstante, la relación y los resultados con las didácticas no es tan evidente. Aunque algunas de las universidades cuentan con una línea o grupo de investigación en didácticas, en los currículos no se presenta concretamente cómo estos influyen en los procesos formativos.

Finalmente, los proyectos curriculares de los programas en Humanidades y Lengua Castellana presentan las prácticas sociales y profesionales como un escenario de impacto social en el que los estudiantes tienen contacto con contextos educativos diversos y ponen en función su propio proyecto de formación. Así, la mayoría de los programas académicos se nutren de diferentes actividades y prácticas orientadas al desarrollo de las habilidades lingüísticas, el gusto por el fenómeno literario y del interés por los hechos comunicativos que enmarcan en la cotidianidad. De ahí que cada una de las prácticas educativas cimiente los propósitos de formación de los programas, los cuales tienen un carácter social y un interés especial por influir positivamente en escenarios de formación escolar, donde sea posible una transformación social ligada al lenguaje y en las que los futuros licenciados resulten imprescindibles para la actualización e innovación del aprendizaje de la lengua materna, de su literatura y de los procesos comunicativos que esta entraña. 


\section{Referencias bibliográficas}

Baeza, M. (2002). De las metodologías cualitativas en investigación científico social. Diseño y uso de instrumentos en la producción de sentido. Concepción: Universidad de Concepción.

Bonilla, E. \& Rodríguez, P. (2000). Más allá del dilema de los métodos. La investigación en ciencias sociales. Bogotá: Universidad de los Andes.

Consejo Nacional de Acreditación. (1999). Pedagogía y educación: reflexión sobre el decreto 272 de 1998 para acreditación previa para programas de Educación. Bogotá: Consejo Nacional de Acreditación CNA.

Eco, U. (1977). Tratado de semiótica general. Barcelona: Lumen.

Gil-Albarellos, S. (2006). Introducción a la literatura comparada. Valladolid: Universidad de Valladolid.

Huaman, M. A. (2007). Fundamentos de la investigación literaria. Tesis. Revista de la Unidad de Post Grado de la Facultad de Letras y Ciencias Humanas de la UNMSM, N 1 , 2007.

Marina, J. A. (1999). La selva del lenguaje. Introducción de los sentidos. Barcelona: Anagrama.

Ministerio de Educación Nacional -MEN-. (2008). Tensiones entre el desarrollo curricular del sistema educativo colombiano y el proceso de construcción cultural afrocolombiana. Disponible en: http://www.colombiaaprende.edu.co/html/mediateca/1607/ articles-174672_archivo.pdf.

Ministerio de Educación Nacional -MEN-. (2012). Política y sistema colombiano de formación y desarrollo profesional de educadores. Bogotá: Ministerio de Educación Nacional. (Sin publicar).

Morin, E. (2005). Introducción al pensamiento complejo. Barcelona: Gedisa.

Nicolescu, B. (2006). Transdiciplinariedad: pasado, presente y futuro. Primera parte. En: Revista Visión Docente Con-ciencia, (31):15-31. Disponible en: http://www.ceuarkos. com/Vision_docente/ revista31/t3.htm. Segunda parte. En: Revista Visión Docente Con-Ciencia, (32):14-33. Disponible en: http://www.ceuarkos. com/Vision_docente/ revista32/t4.htm

Reguera, A. (2008). Metodología en investigación lingüística. Buenos Aires: Brujas.

Sistema Nacional de Acreditación. (2013). Lineamientos para la acreditación de programas de pregrado. Bogotá: Consejo Nacional de Acreditación -CNA-.

Soriano, A., Perdomo, W. \& Sánchez, S. (2014). Alfabetización en el medio cine. El discurso audiovisual en el aula. Bogotá: CMYK. 
Unesco. (1998). Conferencia Mundial sobre la Educación Superior. La educación superior en el siglo XXI: visión y acción. París: Unesco..

Unesco. (2010). Formación de docentes. Disponible en: http://www.unesco.org/new/ es/unesco/themes/icts/teacher-education/ 